\title{
Video Game Device Haptic Interface for Robotic Arc Welding
}

\author{
HSI 2009
}

\author{
Corrie I. Nichol \\ Milos Manic
}

May 2009

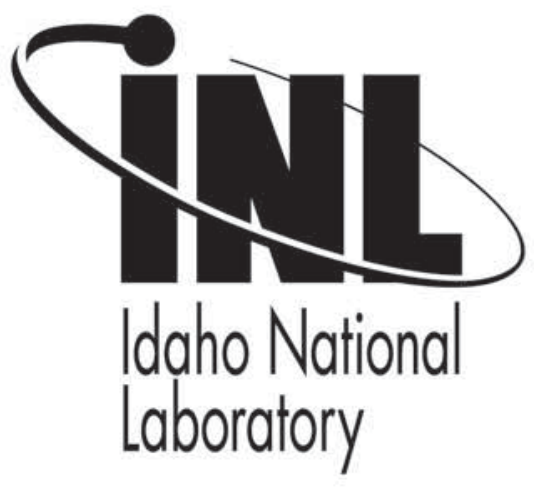

This is a preprint of a paper intended for publication in a journal or proceedings. Since changes may be made before publication, this preprint should not be cited or reproduced without permission of the author. This document was prepared as an account of work sponsored by an agency of the United States Government. Neither the United States Government nor any agency thereof, or any of their employees, makes any warranty, expressed or implied, or assumes any legal liability or responsibility for any third party's use, or the results of such use, of any information, apparatus, product or process disclosed in this report, or represents that its use by such third party would not infringe privately owned rights. The views expressed in this paper are not necessarily those of the United States Government or the sponsoring agency. 


\title{
Video Game Device Haptic Interface for Robotic Arc Welding
}

\author{
Corrie I. Nichol†, Member, IEEE, Milos Manic + , Senior Member, IEEE \\ $\dagger$ Idaho National Laboratory, †University of Idaho Idaho Falls
}

\begin{abstract}
Recent advances in technology for video games have made a broad array of haptic feedback devices available at low cost. This paper presents a bi-manual haptic system to enable an operator to weld remotely using a commercially available haptic feedback video game device for the user interface. The system showed good performance in initial tests, demonstrating the utility of low cost input devices for remote haptic operations.
\end{abstract}

Index Terms-Robot tactile systems, Robots, Ergonomics, Haptics

\section{INTRODUCTION}

$\mathrm{H}$ APTIC systems for remote operations have been widely studied, and have recently been marketed for video games. The commercially available video game control

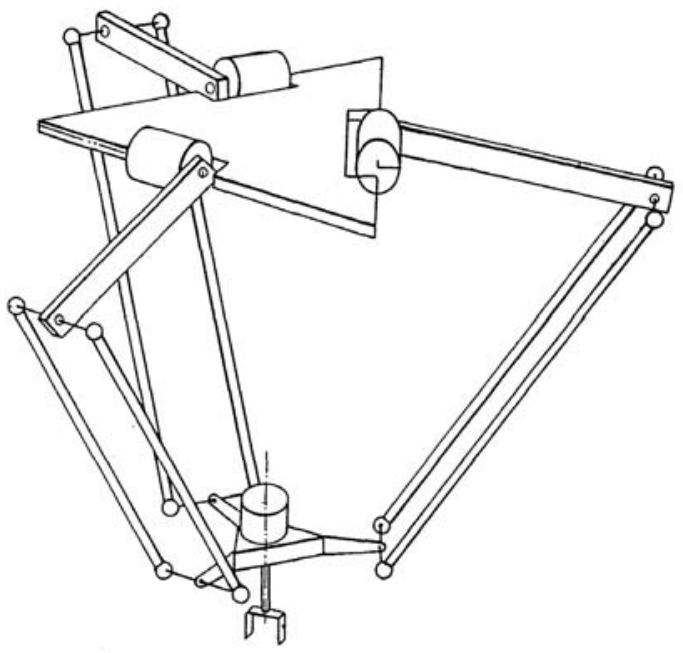

Fig. 1, Delta Robot device [25].

device selected (see Fig. 1) is relatively new, and is an application of the Delta Robot [2]. This device is unique among haptic feedback video game devices in that the input

Work supported through the INL Laboratory Directed Research \& Development (LDRD) Program under DOE Idaho Operations Office Contract DE-AC07-05ID14517.

C. I. Nichol is a Research Engineer at the Idaho National Laboratory, Idaho Falls, ID 83415 USA (phone 208-526-9385, fax 208-526-0690, e-mail: corrie.nichol@inl.gov).

M. Manic is a director of Modern Heuristics Research Group and faculty with the Dept. of Computer Science, University of Idaho Idaho Falls (email: misko@ieee.org). degrees of freedom are linear along the $\mathrm{X}, \mathrm{Y}$, and $\mathrm{Z}$ axes, making the device interesting for robotic work.

Remote haptic interfaces are interesting for a variety of processes for which the environment is hazardous, or inaccessible to human operators. Early work at the Joint European Torus (JET) tokamak (a machine producing a toroidal magnetic field for confining a plasma), yielded the Mascot IV for the end goal of operating in a high radiation field [3]. NASA, in collaboration with DARPA, has been developing the "Robonaut," an anthropomorphic robotic device for extra vehicular activities in space [4]. Telerobotic systems have recently gained a strong presence in the medical community, especially for minimally invasive surgery [5].

Recent work has also focused on stability of a remote haptic feedback system, as evidenced by literature [6-13], much of which has focused on passivity control. Significant amount of work has also focused on making the teleoperation system transparent to the user, including, as described by the work of Flemmer, Lawrence, and Zhu in [12, 14, 15].

Recent work into novel methods for the control of robotic systems has included the work done by Stan et al. [16].

However, there has not been much work done with regards to the use of remote haptic systems for industrial type processes, specifically related to the design and testing of a welding system.

This paper presents the development and initial testing of a system for conducting remote industrial type operations. The work described in this paper is part of an ongoing project focusing on the development of a remote immersion system for welding. The paper continues as follows. Section II describes the hardware part of the presented robotic arc welding system, while Section III presents the software developed for this system. Section IV presents the testing of the developed robotic system, ending with conclusion in Section V.

\section{Robotic ARC Welding HARDWARE SYSTEM}

The presented novel system consists of a collection of commercially available components. The components were chosen both for their availability and price. After initial testing, the final system proved to be both highly functional and cost effective, and demonstrated the state of the art of commercially available products for this type of end application. The collection of hardware described in this section was then combined via developed software (presented in Section III), to accomplish the goal of a remote telepresence 
system. A complete description of the components used follows.

\section{A. Robotic Actuators}

The robotic actuator selected for this project was a commercially available robotic manipulator with 6-DOF (Fig. $3)$. This device is a robotic manipulator with proportions roughly similar to the proportions of a human arm. This makes it ideally suited for remote immersion. (i.e. giving the operator a sense of being physically present in the remote environment) Two of these devices were used to create a remote bi-manual actuator system.

\section{B. Haptic Input Devices}

Force feedback devices have recently become popular as commercial video game devices. One of the most recent, a Delta Robot [2] configuration is a force-controlled parallel robotic device, which provides 3-DOF input and force feedback, along the three principal linear axes, $\mathrm{X}, \mathrm{Y}$, and Z. Several companies sell more elaborate research type haptic equipment, among these is the Quanser $\AA^{1}$ company, who sells a fully integrated remote robotic platform and haptic input device [17].

The motivation for selecting this device for solving of the problem of remote welding was its ubiquitous market

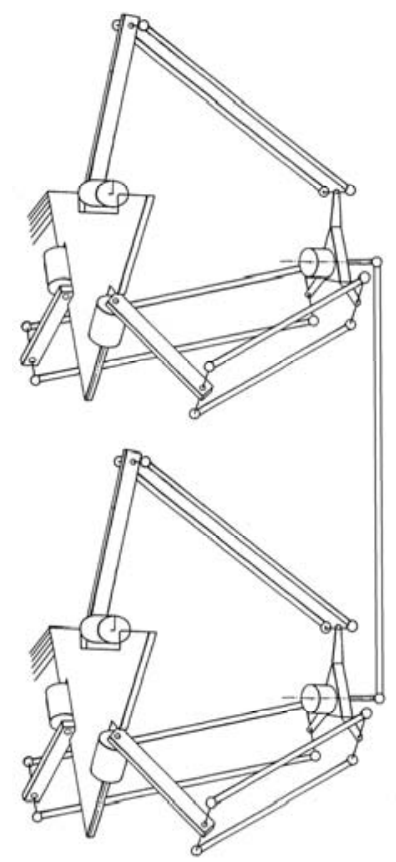

Fig. 2. Two Delta devices for 5 DOF adapted from [2]

presence and affordable price. Because these devices are only capable of 3 input and output DOF, two devices were used together to get a 5-DOF input/output device. By mounting

\footnotetext{
${ }^{1}$ References herein to any specific commercial product, process, or service by trade name, trademark, manufacturer, or otherwise, does not necessarily constitute or imply its endorsement, recommendation, or favoring by the U.S. Government, any agency thereof, or any company affiliated with the Idaho National Laboratory.
}

one device above the other, and connecting the handles of each device with a simple linkage, the differential input and output of the devices along the horizontal plane provides two additional degrees of freedom. The configuration of these devices is shown in Fig. 2. These devices could be further augmented with a rotational actuator and sensor on the handle linkage to give the sixth, and missing degree of freedom.

\section{Force Sensor}

The strategy selected for force sensing on the remote robotic manipulator was to place a force/torque sensor between the end of the robotic manipulator and the tool used by the manipulator. In terms of full remote immersion, the feedback and perception of the operator can also be further augmented by sensing the torques at each robot joint, thereby including any interaction between other links of the robotic manipulator

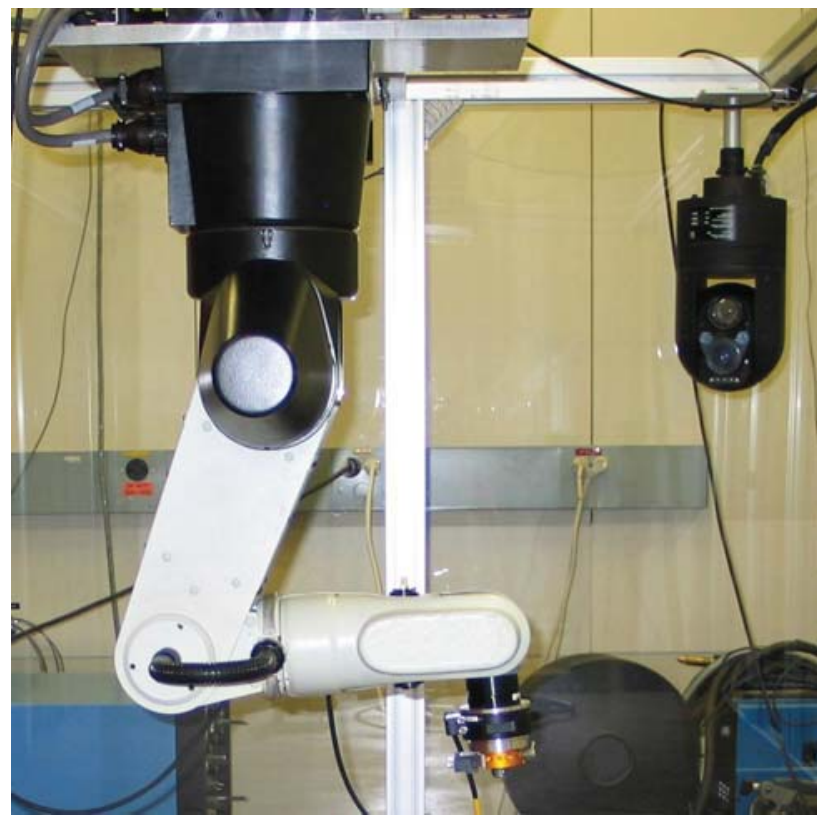

Fig. 3. Robotic Actuator with Force Sensor.

and environment (i.e. bumping something with the robot's "elbow", or any interaction with any part of the robot other than the end effector). However, for the purpose of this system it was deemed sufficient to sense only interaction forces of the remote tool, and to feed back only those forces to the haptic input device.

The sensor selected for this was a commercially available sensor device, which provides full force/torque feedback (i.e. force along the 3 principal orthogonal axes, and torque about each of these axes).

\section{Computer Hardware}

The manufacturer supplied robot controllers were too slow for direct haptic feedback control, so these were replaced with $3^{\text {rd }}$ party motor drives and computer interface control hardware.

\section{E. Remote Visualization}

Operation of a robotic device in a remote environment via a telepresence system requires that the user be provided visual feedback. Visual feedback was provided by a head 
tracking/head aimed viewer (Fig. 4). This system senses the motion of the users head, and uses this information to aim the camera gimbal, allowing the user to look around intuitively in the remote environment without the necessity of some other interface for camera gimbal control.

\section{F. Welding}

One of the stated goals of this project was to develop a remote telepresence system that would enable a trained welder to conduct welds remotely. For this initial work a Gas Metal Arc Welding (GMAW) process was selected because it proved to be significantly easier to produce welds with than
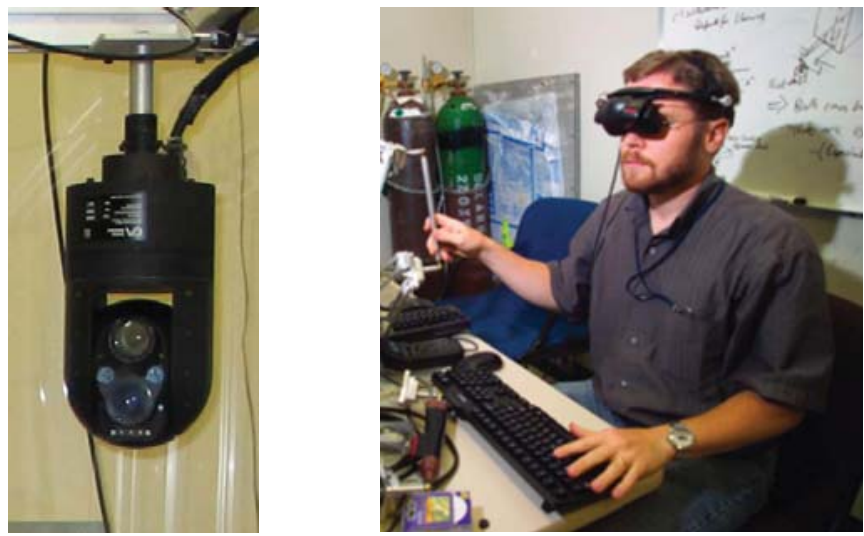

Fig. 4. Camera gimbal and user with visor.

with other welding processes. In order to do this an end effector using a commercial GMAW weld torch was designed and built. The weld torch was attached to a robot tool plate that further attaches to the end of the robotic manipulator.

\section{Robotic Arc Welding Software System}

The collection of hardware described above was then combined via developed software to accomplish the goal of creating a remote telepresence system.

\section{A. Inverse Kinematics}

In order to achieve the correct end effector position and orientation, it was necessary to calculate the relationship between the pose of the input device and the required joint angles. This was also necessary since the input video game devices are not kinematically similar to the robotic manipulators, and because the commercial robotic controllers are not used in the final system.

The common representation of position and orientation between the input devices and the robot was selected to be a position in $\mathrm{X}, \mathrm{Y}$, and $\mathrm{Z}$ coordinates, and the Euler-angle rotations (z, y, z'). Fig. 5 illustrates the euler angle orientations, as described by Ginsberg [1]where the $\mathrm{z}$ above is noted as the precession angle, the $\mathrm{y}$ is noted as the nutation angle, and the final rotation $z^{\prime}$ is the spin angle.

For a given point within the robot working range there are a total of eight candidate solutions possible. Given the set of six joint angles for a six degree of freedom robotic manipulator, the end point and orientation is given by the equation (see [18]):

$$
\bar{T}=\overline{A_{0}} \cdot \overline{A_{1}} \cdot \overline{A_{2}} \cdot \overline{A_{3}} \cdot \overline{A_{4}} \cdot \overline{A_{5}} \cdot \overline{A_{6}}
$$

Here, $\bar{T}$ is the homogeneous transform describing the position and orientation of the last joint frame of the robot relative to the base coordinate frame.

The homogeneous transform:

$$
\overline{A_{x}}, \quad x=1, \ldots, 6
$$

describes the position and orientation of each joint frame, including one for the position and orientation of the joint frame of the tool.

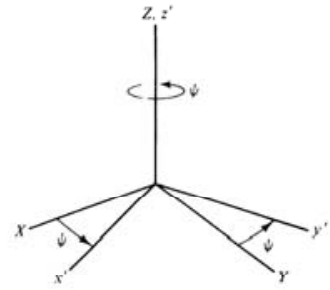

a) Precession.

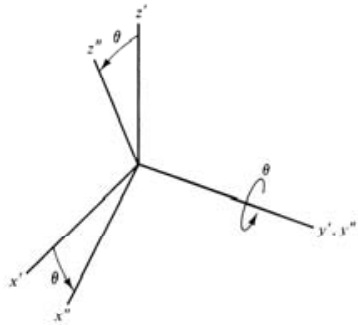

b) Nutation.

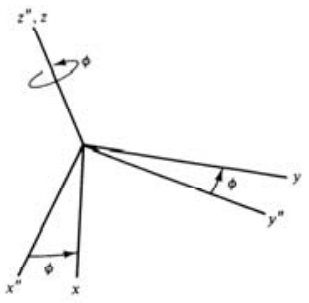

c) Spin.

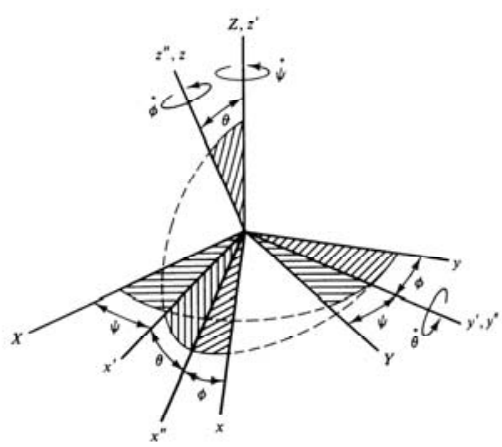

d) Eulerian angles and reference frames.

Fig. 5. Euler Angle Rotation from [1].

The equations to solve for the four candidate solutions to the first three joint angles can be derived from a trigonometric analysis. Each of these solutions are used twice for the final eight candidate solutions. Derivation of the four equations follows from a trigonometric analysis based on the robot's physical configuration. If $\overline{T_{D}}$ describes the desired homogeneous transform for the end of the robotic manipulator, equations for the candidate solutions for the angles of the last three joints can be found by careful selection of parameters in the following three matrix equations (see [18]):

$$
\begin{aligned}
& { }_{3}^{1} T^{-1} \cdot \overline{T_{D}}=\overline{{ }_{6}^{3} T} \\
& { }_{4}^{1} T^{-1} \cdot \overline{T_{D}}=\overline{{ }_{6} T} \\
& { }_{5}^{1} T^{-1} \cdot \overline{T_{D}}=\overline{A_{6}}
\end{aligned}
$$

where

$$
\overline{{ }_{x}^{a} T}=\overline{A_{a}} \cdot \overline{A_{a+1}} \cdot \overline{A_{a+2}} \cdot \ldots \cdot \overline{A_{x}}
$$

Where $a$ and $x$ represent specific joint numbers, so ${ }_{6}^{3} T$ represents the homogeneous transform from joint 3 to joint 6 
of the robotic manipulator.

The resulting set of equations to solve the eight candidate solutions can easily be programmed and solved for each time step during operation. Inclusion of an extra homogeneous transform to describe the geometry of the tool attached to the end of the robot is a simple exercise.

It is then necessary to eliminate candidate solutions that contain joint angles outside the range of motion of the robot, and to then select from the remaining candidate solutions which solution will be used. This was accomplished by assigning a weight to each of the eight candidate solutions. These weights were assigned as follows.

$$
W_{i}=W_{\text {curr }} \cdot\left(\sum_{j=1}^{6}\left|\theta_{i, j}-\theta_{j}^{\prime}\right|\right)+W_{\lim }
$$

where

$W_{\text {curr }}$ is the weight to bias to the current solution

$W_{i}$ is the weight for candidate solution $i(i=1,2, \ldots, 8)$

$j$ is the joint number $(j=1,2, \ldots, 6)$

and $W_{\text {lim }}$ is selected to exclude solutions which exceed the joint limits of the robotic manipulator.

Candidate solutions were first checked against the physical joint limits of the manipulator. Solutions with angles outside the physical limitations of the robot were given a very high weight ( $\left.W_{\lim }=10,000\right)$ to exclude them from selection. The remaining candidate solution joint angles were then compared to the current pose of the robot. The magnitude of the difference between each candidate joint angle and the current joint angle were summed to get the weight for that candidate solution. In equation 4 above, the candidate joint solutions are indexed by $i$ and angles of each of the individual joints are indexed by $j$, i.e. $\theta_{i, j}$ indicates the angle of joint $j$ for the candidate solution $i$ and $\theta_{j}^{\prime}$ indicates the angle of joint $j$ selected on the previous iteration. To further bias the solution method to the current solution (and thereby avoid unnecessary joint configuration changes which could result in erratic and potentially dangerous motion of the robot), the candidate solutions that were not selected on the previous iteration were multiplied by a biasing factor $W_{\text {curr }}$, where $W_{\text {curr }}=1$ for the previously selected solution, and $W_{\text {curr }}=\alpha$ for all other candidate solutions, where $\alpha>1$ to bias the solution to the previously selected joint configuration solution. The candidate solutions are then compared based on the weight factors calculated, and the candidate solution with the lowest weight $W_{i}$ is selected. The end result is that the robot will usually only change to an alternate solution when some joint angle is exceeded.

\section{B. Input Device Kinematics}

The haptic feedback and input device reports the X,Y,Z position, and receives as an input the desired forces along each of the principal axes. By joining two devices it is necessary to use the position of each device to derive the two angles and three positions desired. Angles are calculated by comparing the difference of each device axis. The vertical separation between the input devices is known to be the handle length.

These angles are then converted to the Euler angles for input into the robotic manipulator inverse kinematics routine. Because there is no actuator or sensor for the rotation, the rotation was set to zero for all calculations.

\section{Force Feedback and Control Strategy}

The robot position is commanded to mirror the position of the manipulator, and the forces encountered by the sensor on the robotic manipulator are then fed back into the haptic feedback and input device. This is often referred to as a position command force feedback haptic control strategy.

\section{Initial Intervention Strategies}

Because the input device (the pair of haptic feedback and input devices) has a rather limited range of motion compared to the robotic manipulator, the motion of the input device in the $\mathrm{X}, \mathrm{Y}$, and $\mathrm{Z}$ axes was scaled up by a factor of two, allowing a greater range of motion of the robotic manipulator.

The results of scaling the forces encountered by the robotic manipulator were also investigated, in effect, making the user more sensitive to forces in the remote environment. It was found that the forces could be scaled up by a factor of two without significantly impairing the overall system performance.

\section{Testing of Robotic Arc Welding System}

The review of testing of robotic arc welding system will be elaborated with regards to the system stability, initial welding test, and finally analysis of initial weld test results.

System stability was investigated on several test examples. Early qualitative testing was conducted by placing objects of different stiffness in the remote manipulator work cell. Interaction with relatively soft objects gave good performance. The manipulator was able to track motion accurately, and the interaction showed no contact instability (commonly referred to as "haptic bounce" or "chatter"). These objects included a phone book, a cardboard box and a basketball.

The device was then used to push against a steel plate. During fast impact with the steel plate the system exhibited some contact instability. As expected, this instability, or bounce effect, was more pronounced with larger motion scaling, and increased force sensitivity.

Next, initial welding test of the presented robotic arc welding system was performed. For comparison, Fig. 6a shows a weld on a flat plate conducted by hand (i.e. the operator held the weld torch). Fig. $6 \mathrm{~b}$ shows a fillet weld made with the haptic system in which the operator had the weld torch too far away from the joint that was to be welded, and also welded over an earlier bad weld, illustrating some of the limitations of the remote camera system discussed below. The weld in Fig. 6c represents the last and best weld of the 
series.

The initial weld test shows that a weld of acceptable quality can be made remotely via the system. All of the welding

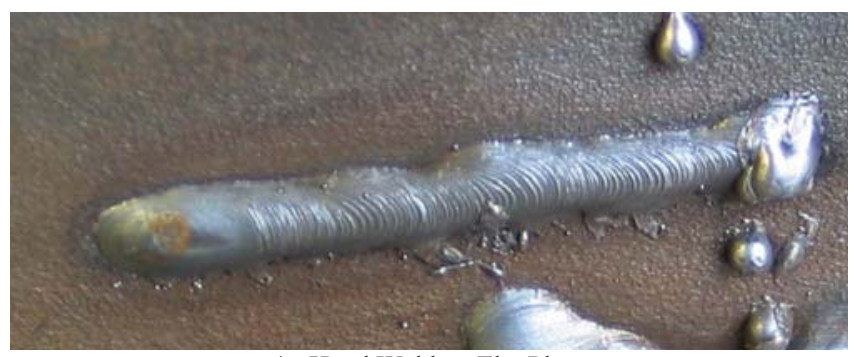

a). Hand Weld on Flat Plate.

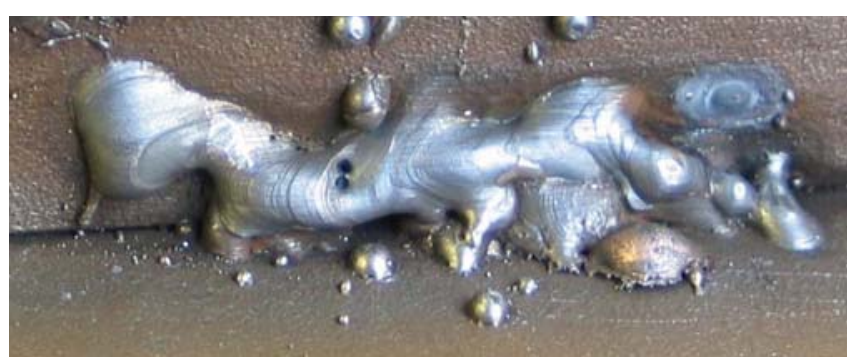

b). Early Fillet Weld.

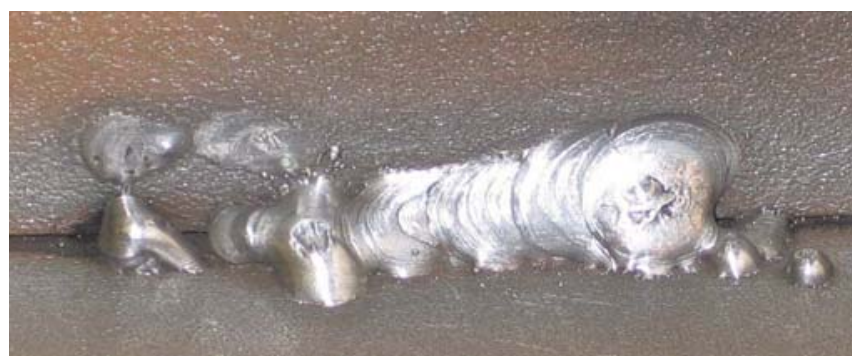

c). Final Test Weld.

Fig. 6. Test Welds

conducted for the initial trial was conducted by an operator with no formal weld training, so any limitation in weld quality is equally likely to be a limitation of the operator's skill set as a limitation of the remote immersion system. Further testing will include trained welders.

Several limitations were exposed during the initial testing. The vision system was incapable of visualizing the weld during welding. This was an issue that has been considered by many researchers and arc viewing camera producers. The cameras needed to visualize the weld pool and arc were insufficient to visualize a normal environment. Future work will include the inclusion of commercial arc viewing cameras for better weld visualization, and a strategy to switch the view provided to the viewer in such a way that the user is able to receive all necessary visual information.

The user, via the current head aimed remote viewer, has little depth perception. Vision feedback is monocular. This provided only a moderate limitation in the actual performance of the weld (see Fig. 6b), as the user was able to tap the surfaces of the weld prep. (the joint to be welded), then back off a prescribed distance prior to initiation of a weld. This would also have been less of an issue with the appropriate welding arc viewing camera equipment described above, and placed at the appropriate angle to the weld. This will be investigated to determine how to optimally provide this information to the user.

Lastly, the robotic manipulator is capable of a significantly greater range of motion than was allowed by the input device. Future work will include investigation of strategies to expand the effective working range of the input device, including more sophisticated motion scaling strategies.

\section{CONCLUSION}

The design of a low cost remote haptic system using commercially available haptic feedback video game device was presented in this paper. The initial viability for remote haptic control of a robotic manipulator was demonstrated and discussed. The haptic feedback system was successfully tested on performing remote welding operations. The experimental results were illustrated by attached photos.

Further work includes testing with professional welders and improvements with regards to the "haptic bounce" or "chatter" reduction and introduction of improved vision system.

\section{REFERENCES}

[1] J. H. Ginsberg, Advanced Engineering Dynamics, 2nd ed. Cambridge, UK: Cambridge University Press, 1998.

[2] R. Clavel, "Device for the Movement and Positioning of an Element in Space", U.S. Patent Number: 4976582

[3] L. Galbiati, T. Raimondi, P. Garetti, and G. Costi, "Control and Operational Aspects of the Mascot-4 Force Feedback Servomanipulator of JET," in 14th IEEE / NPSS Symposium on Fusion Engineering, San Diego, Ca, 1991, pp. 563-566.

[4] E. D. Flinn, "Robonauts join "spacewalk squad"," Aerospace America, vol. 41, pp. 18-19, Oct 2003.

[5] A. M. Okamura, "Methods for haptic feedback in teleoperated robot-assisted surgery," Industrial Robot-an International Journal, vol. 31, pp. 499-508, 2004.

[6] Y. Ye and P. Ya-Jun, "A Simplified Time Domain Passivity Control of Haptic Interfaces," in Proceedings of the 11th IASTED International Conference, Intelligent Systems and Control (ISC 2008), Orlando, Florida, USA, 2008.

[7] R. W. Daniel and P. R. McAree, "Fundamental limits of performance for force reflecting teleoperation," International Journal of Robotics Research, vol. 17, pp. 811-830, Aug 1998.

[8] R. Cortesao, J. Park, and O. Khatib, "Real-time adaptive control for haptic manipulation with active observers," in IEEE/RSJ International Conference on Intelligent Robots and Systems, Las Vegas, Nv, 2003, pp. 2938-2943.

[9] R. Cortesao, J. Park, and O. Khatib, "Real-time adaptive control for haptic telemanipulation with Kalman active observers," IEEE Transactions on Robotics, vol. 22, pp. 987-999, Oct 2006.

[10] K. Krishnaswamy and P. Y. Li, "Bond graph based approach to passive teleoperation of a hydraulic backhoe," in ASME International Mechanical Engineering Congress, Anaheim, CA, 2004, pp. 176-185.

[11] G. Niemeyer and J. J. E. Slotine, "Stable Adaptive Teleoperation," IEEE Journal of Oceanic Engineering, vol. 16, pp. 152-162, Jan 1991.

[12] D. A. Lawrence, "Stability and Transparency in Bilateral Telepoeration," IEEE Transactions on Robotics and Automation, vol. 9, pp. 624-637, Oct 1993.

[13] S. H. Dandach and S. Dasgupta, "Optimal design of stable haptic interfaces," in SICE 2004 Annual Conference, Sapporo, JAPAN, 2005, pp. 1083-1087. 
[14] H. Flemmer, B. Eriksson, and J. Wikander, "Control design for transparent teleoperators with model parameter variation," in 19th IEEE International Conference on Robotics and Automation (ICRA), Washington, Dc, 2002, pp. 2956-2961.

[15] M. Zhu and S. E. Salcudean, "Achieving Transparency for Teleoperator Systems Under Position and Rate Control," in 1995 IEEE/RSJ International Conference on Intelligent Robots and Systems - Human Robot Interaction and Cooperative Robots, Pittsburgh, Pa, 1995, pp. 7-12.

[16] S. D. Stan, M. Manic, V. Maties, and R. Balan, "A Novel Virtual Reality Robot Interface for Isoglide3 Parallel Robot," in lst International Conference Intelligent Robotics and Applications, Wuhan, PEOPLES R CHINA, 2008, pp. 1265-1275.

[17] Quanser ${ }^{\circledR}$, (URL from Jan., 2009),

http://www.quanser.com/NET/Industrial/Systems_and_Products/Pr od 5Dof Haptic.aspx

[18] B. Armstrong, O. Khatib, and J. Burdick, "The explicit dynamic model and inertial parameters of the Puma 560 arm," in Proceedings IEEE International Conference on Systems, Man, \& Cyberetics, 1986, pp. 510-518. 